\title{
„Was man nicht messen kann, kann man nicht verbessern"
}

\section{Die Bedeutung der Surveillance zur Qualitätssicherung}

\author{
Christine Geffers, Elisabeth Meyer, Heinz-Michael Just
}

Laut Weltgesundheitsorganisation sind Antibiotikaresistenz und nosokomiale Infektionen eines der größten Gesundheitsprobleme weltweit. In den $27 \mathrm{EU}$ Mitglieds-Ländern Europas geht man von 4 Millionen Patienten aus, die jährlich eine nosokomiale Infektion erleiden mit etwa 37000 dadurch bedingten Todesfällen.

Der britische Physiker Lord Kelvin stellte fest: „Wenn man messen kann, worüber man spricht und es in Zahlen ausdrücken kann, dann weiß man etwas darüber. Wenn man es nicht in Zahlen ausdrücken kann, dann ist das Wissen dürftig und unzureichend."

Oder einfach ausgedrückt: Wir brauchen Zahlen und Fakten, um vernünftig über eine Sache sprechen zu können. Unser gefühltes Wissen über Dinge reicht nicht aus, um Probleme zu erkennen und nachfolgend Lösungsansätze zu finden.

Auf diesem Grundprinzip der modernen Wissenschaft fußt auch das Prinzip der Surveillance - egal, ob es sich um Surveillance von nosokomialen Infektion, Antibiotikaeinsatz oder von multiresistenten Erregern handelt. Gerade im Zusammenhang mit Infektionen sind Daten zur Epidemiologie ein substantielles Werkzeug zur Prävention. Die Surveillance liefert die dafür unabdingbar benötigten Daten für einzelne Bereiche, Einrichtungen und Krankenhäuser. Der Begriff Surveillance meint dabei allerdings sehr viel mehr als die bloße Registrierung von Ereignissen. Unter dem Begriff werden das Erheben, aber auch die Bewertung und das Übermitteln der Erkenntnisse an die Vor-OrtTätigen verstanden. Den Hygienemitar- beitern wird ebenso wie dem anderen medizinischen Personal damit die Möglichkeit gegeben, den Erfolg ihrer Präventionsbemühungen beurteilen zu können und gegebenenfalls die Bemühungen zu intensivieren oder neue Präventionsstrategien zu etablieren.

Allgemein ist das Problem bei der Prävention, dass der Erfolg üblicherweise nicht direkt sichtbar ist: eine nicht erlittene Erkrankung zeigt sich nicht unmittelbar. Dadurch wird der Sinn teilweise aufwendiger Präventionsmaßnahmen im klinischen Alltag häufig in Frage gestellt oder auch bewusst oder unbewusst deren Umsetzungsgrad reduziert. Die Surveillance spielt gerade hierbei eine entscheidende Rolle, da sie nicht nur über die reine Sammlung von Daten funktioniert. Die Erhebung auf den Stationen bzw. in den Einrichtungen bringt das Problem nosokomialer Infektionen in das Bewusstsein der Mitarbeiter. Die Kommunikation zwischen Hygiene, Pflege und Ärzten wird gefördert und auf eine belastbare Basis gestellt. Aber noch vor allem anderen wird durch die Surveillance hygienisches Handeln mit nosokomialen Infektionen sichtbar und nachvollziehbar in Verbindung gebracht.

International ist die Surveillance als Grundvoraussetzung aller Präventionsstrategien längst anerkannt. Am 27. und 28. November 2012 tagte das ECDC (Europäisches Center for Disease Control and Prevention) in Berlin, mit dem Ziel, Surveillance europaweit zu standardisieren, weiter zu optimieren und auszudehnen.
Die renommierten CDC in den USA bzw. das ECDC in Europa, sowie in Deutschland das RKI erachten die Surveillance als allgemein akzeptierte und unabdingbare Methode, um nosokomiale Infektionen zu reduzieren. Der Gesetzgeber hat dies bereits vor Jahren erkannt und im Infektionsschutzgesetz aus dem Jahr 2001 die Durchführung einer Surveillance in allen Krankenhäusern in Deutschland verpflichtend festgelegt.

Deutschland nimmt dabei europaweit und international eine viel beachtete Vorreiterrolle ein. Das vom Nationalen Referenzzentrum für die Surveillance von nosokomialen Infektionen betriebene Krankenhaus-Infektions-SurveillanceSystem (KISS) ist weltweit das zweitgrößte Surveillance-System nach dem US-amerikanischen System.

Mehr als 1000 Krankenhäuser beteiligen sich freiwillig daran und liefern nach einheitlicher Methodik und individuell in unterschiedlichem Umfang Daten zur Häufigkeit von nosokomialen Infektionen in Risikobereichen, zu Antibiotikaverbräuchen, resistenten Erregern und Händedesinfektionsmittelverbräuchen. Damit wird es von der Mehrheit der deutschen Krankenhäuser als wichtiges Instrument der Qualitätssicherung genutzt und geschätzt. Inzwischen beteiligen sich auch aus anderen Ländern Europas Einrichtungen an KISS und die Methodik des KISS bildete das Vorbild beim Aufbau anderer internationaler Systeme. Eine Analyse des KISS wie auch anderer Surveillance-Syste- 
me haben mehrfach den Nachweis erbracht, dass durch die Surveillance und das Lernen aus Surveillance-Daten Infektionsraten positiv zu beeinflussen sind.

Aber natürlich braucht sorgsame Surveillance wie alle medizinischen Maßnahmen auch Zeit und erfordert Aufwand. Dass diese Investitionen in die gesetzlich geforderte Etablierung einer Surveillance - und KISS hat hierzu maßgeblich beigetragen in Deutschland sinnvoll investiert ist, lässt sich nicht nur an den in Peer-review-Journals veröffentlichten Reduktionserfolgen bei Analyse der Infektionsraten während der Beteiligung am KISS erkennen. Der Erfolg zeigt sich auch daran, dass die nosokomialen Infektionen in Deutschland in den letzten 17 Jahren entgegen anderslautender Darstellungen in der Laienpresse nicht angestiegen sind.Zur Erinnerung: die erste repräsentative Erhebung vor 17 Jahren (NIDEP I) zeigte, dass bei 3,5\% aller Patienten eine Krankenhausinfektion zum Untersuchungszeitraum festzustellen war und diese Zahl ist aktuell in der repräsentativen Punktprävalenzstudie aus 2011 etwa gleich geblieben. Dies trotz der enormen und zunehmend invasiven Fortschritte in der Medizin, dem auch im Krankenhaus festzustellenden demografischen Wandel bei gleichzeitiger Reduktion/
Rationalisierung von personellen Ressourcen und einer zunehmenden Verkürzung der Aufenthaltsdauer, sowie einer überproportionalen Anwesenheit von Risikound nosokomial infizierten Patienten was bei Prävalenzerhebungen eher zu einer Überschätzung führt.

Die neuen Daten der Punktprävalenzstudie aus 2011 unterstreichen den Erfolg, der nur durch die Zusammenarbeit von engagierten Klinkern und Hygienefachpersonal möglich war und ist. Surveillance kostet nicht nur Zeit, sie macht auch Erfolg sichtbar!

Literatur 1 Gesetz zur Änderung des Infektionsschutzgesetzes. Bundesgesetzblatt Teil I Nr. 412011

2 Gastmeier P, Behnke M, Breier AC et al. [Healthcare-associated infection rates: measuring and comparing : Experiences from the German national nosocomial infection surveillance system (KISS) and from other surveillance systems]. Bundesgesundheitsblatt Gesundheitsforschung Gesundheitsschutz2012; 55: $1363-1369$

3 Gastmeier P, Schwab F, Behnke M et al. Decreasing healthcare-associated infections
(HAI) is an efficient method to decrease healthcare-associated Methicillin-resistant S. aureus (MRSA) infections Antimicrobial resistance data from the German national nosocomial surveillance system KISS. Antimicrob Resist Infect Control2012; 26: 1 -3

4 Schwab F, Gastmeier P, Piening B et al. The step from a voluntary to a mandatory national nosocomial infection surveillance system: the influence on infection rates and surveillance effect. Antimicrob Resist Infect Control2012; 1: 24

5 Gastmeier P, Schwab F, Chaberny I et al. Individual units rather than entire hospital as the basis for improvement: the example of two Methicillin resistant Staphylococcus aureus cohort studies. Antimicrob Resist Infect Control2012; 1: 8

6 Behnke M, Gastmeier P, Geffers C et al. Establishment of a national surveillance system for alcohol-based hand rub consumption and change in consumption over 4 years. Infect Control Hosp Epidemiol2012; 33: 618-620

7 Zuschneid I, Rücker G, Schoop R et al. Representativeness of the surveillance data in the intensive care unit component of the German nosocomial infections surveillance system. Infect Control Hosp Epidemiol2010; 31 : 934 938

8 Gastmeier P, Sohr D, Schwab F et al. Ten years of KISS: the most important requirements for success. J Hosp Infect2008; 70: 11 - 16

9 Gastmeier P, Geffers C, Brandt C et al. Effectiveness of a nationwide nosocomial infection surveillance system for reducing nosocomial infections. J Hosp Infect2006; 64: 16-22 